\title{
Genome-wide identification of Bacillus subtilis Zur-binding sites associated with a Zur box expands its known regulatory network
}

\author{
Eric Prestel ${ }^{1,2}$, Philippe Noirot ${ }^{1,2}$ and Sandrine Auger ${ }^{1,2^{*}}$
}

\begin{abstract}
Background: The Bacillus subtilis Zur transcription factor recognizes a specific DNA motif, the Zur box, to repress expression of genes in response to zinc availability. Although several Zur-regulated genes are well characterized, a genome-wide mapping of Zur-binding sites is needed to define further the set of genes directly regulated by this protein.

Results: Using chromatin immunoprecipitation coupled with hybridization to DNA tiling arrays (ChIP-on-chip), we reported the identification of 80 inter- and intragenic chromosomal sites bound by Zur. Seven Zur-binding regions constitute the Zur primary regulon while 35 newly identified targets were associated with a predicted Zur box. Using transcriptional fusions an intragenic Zur box was showed to promote a full Zur-mediated repression when placed within a promoter region. In addition, intragenic Zur boxes appeared to mediate a transcriptional cis-repressive effect (4- to 9-fold) but the function of Zur at these sites remains unclear. Zur binding to intragenic Zur boxes could prime an intricate mechanisms of regulation of the transcription elongation, possibly with other transcriptional factors. However, the disruption of zinc homeostasis in $\Delta z$ zur cells likely affects many cellular processes masking direct Zur-dependent effects. Finally, most Zur-binding sites were located near or within genes responsive to disulfide stress. These findings expand the potential Zur regulon and reveal unknown interconnections between zinc and redox homeostasis regulatory networks.
\end{abstract}

Conclusions: Our findings considerably expand the potential Zur regulon, and reveal a new level of complexity in Zur binding to its targets via a Zur box motif and via a yet unknown mechanism that remains to be characterized.

Keywords: Zur regulator, B. subtilis, ChIP-on-chip, Zinc homeostasis, Disulfide stress

\section{Background}

Zinc is an essential trace element for all forms of life. It serves as structural scaffold for protein folding and as cofactor for many enzymes and DNA-binding proteins $[1,2]$. However, due to its toxicity, mechanisms for zinc acquisition and efflux are tightly regulated according to metal ion requirements [3-5].

In the Gram positive bacterium Bacillus subtilis, transcription of genes involved in zinc homeostasis is regulated by Zur, a metalloprotein that binds $\mathrm{Zn}(\mathrm{II})$ as corepressor [6-9]. In vivo, Zur forms a homodimer that binds to a conserved DNA motif, the Zur box, which

\footnotetext{
* Correspondence: sandrine.auger@jouy.inra.fr

${ }^{1}$ INRA, UMR1319 Micalis, F-78352 Jouy-en-Josas, France

${ }^{2}$ AgroParisTech, UMR Micalis, F-78352 Jouy-en-Josas, France
}

overlaps the $\sigma^{\mathrm{A}}$-type promoter elements in target genes [10]. DNA-binding studies demonstrated that Zur requires a minimal 9-1-9 inverted repeat motif for highaffinity binding [11]. A stepwise activation model predicts that Zur may respond to a wide range of intracellular $\mathrm{Zn}$ (II) concentrations to gradually repress the Zur regulon [12]. Zur represses expression of genes encoding a high-affinity zinc ABC transporter ZnuACB [7], a putative low affinity zinc uptake system YciBC [10], a GTP cyclohydrolase IB involved in folate biosynthesis FolEB [13], and zinc-independent alternative ribosomal proteins (RpmEB, RpmGC, and RpsNB) [14-18]. In B. subtilis, zinc is also imported by the P-type ATPase ZosA, whose expression is controlled by the peroxide-sensing regulator, PerR [19]. Induction of ZosA in response to hydrogen peroxide stress leads to $\mathrm{Zn}(\mathrm{II})$ uptake, which 
plays an important protective role against oxidative stress damage [19]. Both ZosA and ZnuACB zinc transporters are involved in the competence developmental process [20]. Zinc homeostasis is also maintained in B. subtilis thanks to a zinc-inducible efflux pump CzcD important for growth in the presence of high concentration of $\mathrm{Zn}$ (II) $[5,21]$. Expression of this system is regulated at the transcriptional level by the metalloregulator CzrA [5].

Despite knowledge of Zur-mediated regulation of zinc homeostasis, a global identification of the genes directly under Zur control is still missing. Here, we used chromatin immunoprecipitation of Zur-DNA complexes coupled with hybridization of DNA to tiled oligonucleotides arrays (ChIP-on-chip) to identify regions enriched for Zur DNA-binding sites in vivo, at the genomic scale. We provide evidence that Zur binds to 80 regions on the chromosome, including previously known promoter regions of the Zur primary regulon as well as a number of inter- and intragenic regions. Half of the newly identified binding sites is associated with a predicted Zur box. We showed that an intragenic Zur box was functional to mediate a Zur-dependent repression when inserted in a promoter region. In addition, several intragenic Zur boxes were able to act as transcriptional cis-repressive element but the direct role of Zur at intragenic sites remains unclear. Finally, our study suggests that Zur binding to newly identified targets could be primed to fine-tune gene expression in interplay with other transcription factors in response to specific conditions such as the disulfide stress.

\section{Results}

\section{C-terminally SPA-tagged Zur is a functional regulator}

The B. subtilis chromosome was modified at the zur locus to express Zur fused at its C-terminus with the SPA tag (Zur $\left.{ }^{\mathrm{SPA}}\right)$. In the resulting zur::zur-spa strain, the $\mathrm{Zur}^{\mathrm{SPA}}$ protein is under the control of its native expression signals. To examine the activity of the $\mathrm{Zur}^{\mathrm{SPA}}$ fusion protein, the $y c i C$ promoter region was fused with the lac $Z$ reporter gene and introduced at the $a m y E$ locus in wild-type, zur::zur-spa and $\Delta z u r: a p h A 3$ strains (Methods) (Table 1). Expression of $y c i C$, monitored by measuring the $\beta$-galactosidase activities, was repressed in wild-type and zur::zur-spa cells ( $\beta$-galactosidase activity $\leq 5 \mathrm{UE}$ ) whereas it was increased by a 160 -fold factor in $\Delta z u r$ cells. Thus, $\mathrm{Zur}^{\mathrm{SPA}}$ functionally repressed $y c i C$ expression as wild-type Zur.

We further tested the effect of ion starvation on $y c i C$ lac $Z$ expression in the same genetic backgrounds. The strains were cultivated in MS medium and samples were spread onto solid medium containing X-gal. A drop of $100 \mu \mathrm{M}$ EDTA, an ion chelating agent, was deposited at the center of the plates. After incubation, a characteristic blue ring was observed around the EDTA drop
Table 1 B. subtilis strains used in this study

\begin{tabular}{|c|c|c|}
\hline Strain & Genotype $^{a}$ & Source \\
\hline BSB1 & $\operatorname{trp}^{+}$ & (Nicolas et al., [26] \\
\hline BSAS36 & zur::zur-spa erm & This work \\
\hline BSAS39 & amyE::pyciC'-lacZ cat & This work \\
\hline BSAS44 & amyE::pyciC-lacZ cat zur-spa erm & This work \\
\hline BSAS45 & $\Delta$ zur::aphA3 & This work \\
\hline BSAS49 & amyE::pyciC'-lacZ cat $\Delta$ zur::aphA3 & This work \\
\hline BSAS225 & amyE::pAymaD'-lacZ cat & This work \\
\hline BSAS331 & amyE::pBymaD'-lacZ cat & This work \\
\hline BSAS227 & amyE::pAydeO'-lacZ cat & This work \\
\hline BSAS332 & amyE::pBydeO'-lacZ cat & This work \\
\hline BSAS229 & amyE::pAywhC'-lacZ cat & This work \\
\hline BSAS333 & amyE::pBywhC-lacZ cat & This work \\
\hline BSAS239 & $\Delta y m a D:: a p h A 3$ & This work \\
\hline BSAS240 & amyE::pAktrD'-lacZ cat & This work \\
\hline BSAS329 & amyE::pBktrD'-lacZ cat & This work \\
\hline BSAS249 & amyE::pAyrpE'-lacZ cat & This work \\
\hline BSAS250 & amyE::pAyrpE'-lacZ cat $\Delta$ zur::aphA3 & This work \\
\hline BSAS251 & amyE::pByrpE'-lacZ cat & This work \\
\hline BSAS252 & amyE::pByrpE'-lacZ cat $\Delta$ zur::aphA3 & This work \\
\hline BSAS296 & $\Delta s p x:: s p c$ & This work \\
\hline BLUC201 & amyE::pAymaD'-luc cat & This work \\
\hline BLUC204 & amyE::pAymaD'-luc cat $\Delta s p x: s p c$ & This work \\
\hline BLUC205 & amyE::pAymaD'-Iuc cat $\Delta$ zur::aphA3 & This work \\
\hline BLUC202 & amyE::pBymaD'-Iuc cat & This work \\
\hline BLUC205 & amyE::pBymaD'-luc cat $\Delta$ zur::aphA3 & This work \\
\hline BLUC227 & amyE::pBymaD'-lacZ cat $\Delta$ spx::spc & This work \\
\hline
\end{tabular}

${ }^{a}$ cat, $\mathrm{pC} 194$ chloramphenicol acetyl-transferase gene; aphA3, Enterococcus faecalis kanamycin-resistance gene; erm, erythromycin-resistance gene; $s p c$, Staphylococcus aureus spectinomycin-resistance gene.

(Additional file 1: Figure S1) indicating that ion deficiency induced $y c i C$ expression in cells synthesizing Zur as well as $\mathrm{Zur}^{\mathrm{SPA}}$ proteins. The binding of $\mathrm{Zur}^{\mathrm{SPA}}$ to the $y c i C$ promoter region was reversible upon metal ion starvation. We concluded from this data that the $\mathrm{Zur}^{\mathrm{SPA}}$ fusion protein was functional for transcriptional regulation.

\section{Genome-wide mapping of Zur binding sites}

To identify Zur-binding targets in the B. subtilis genome, we carried out ChIP-on-chip experiments. The zur::zur-spa strain was grown in LB medium to exponential phase. After cross-linking, Zur-bound DNA was immunoprecipitated using a SPA tag specific antibody. The signals from two independent experiments were processed and peaks corresponding to Zur ${ }^{\mathrm{SPA}}$ binding sites were selected using a cut-off value of 4.0 (see Methods). Overall 80 enriched DNA regions were identified from the 
ChIP-on-chip signals (Additional file 2: Table S1). Among them, 5 were located in genomic regions known to belong to the Zur regulon (Figure 1A) [22]. As in parallel, we used the ChIP-on-chip methodology to study various $B$. subtilis transcription factors $[23,24]$, we observed that all the peaks detected with Zur were exclusively specific to this regulator.

We further compared our data with previous in silico studies [25]. The promoter-proximal Zur-binding sites upstream of $y r p E$ and the pseudogene $S 903$ (also named rpmGC) exhibited Zur box motifs. We then tested expression of the $y r p E$ gene in wild-type and $\Delta z u r$ cells by using a transcriptional fusion between the $y r p E$ promoter region and the lac $Z$ reporter gene. Expression of the PyrpE promoter was 30 -fold increased in a zur mutant compared to the wild-type during the exponential phase of growth (Additional file 1: Figure S1). These results demonstrated the Zur-dependent regulation of $y r p E$. We also detected Zur binding upstream of the short $y c z L$ gene, encoding a protein of unknown function. Expression of $y c z L$ is coregulated with $y c i C$ and is driven from a single promoter leading to the $y c z L y c i C$ transcript [26]. Altogether, these findings delineated the Zur primary regulon, which is now composed of 11 genes expressed from 7 distinct promoters (znuABC, folEByciB, rpsNB, rpmEB, rpmGC, yczLyciC and yrpE) fulfilling three criteria: (i) in vivo Zur binding in ChIP-on-chip experiments; (ii) presence of a Zur box; (iii) Zur-dependent regulation of expression.

In addition, 37 additional promoter-proximal Zur binding sites were detected less than 200 base-pairs upstream

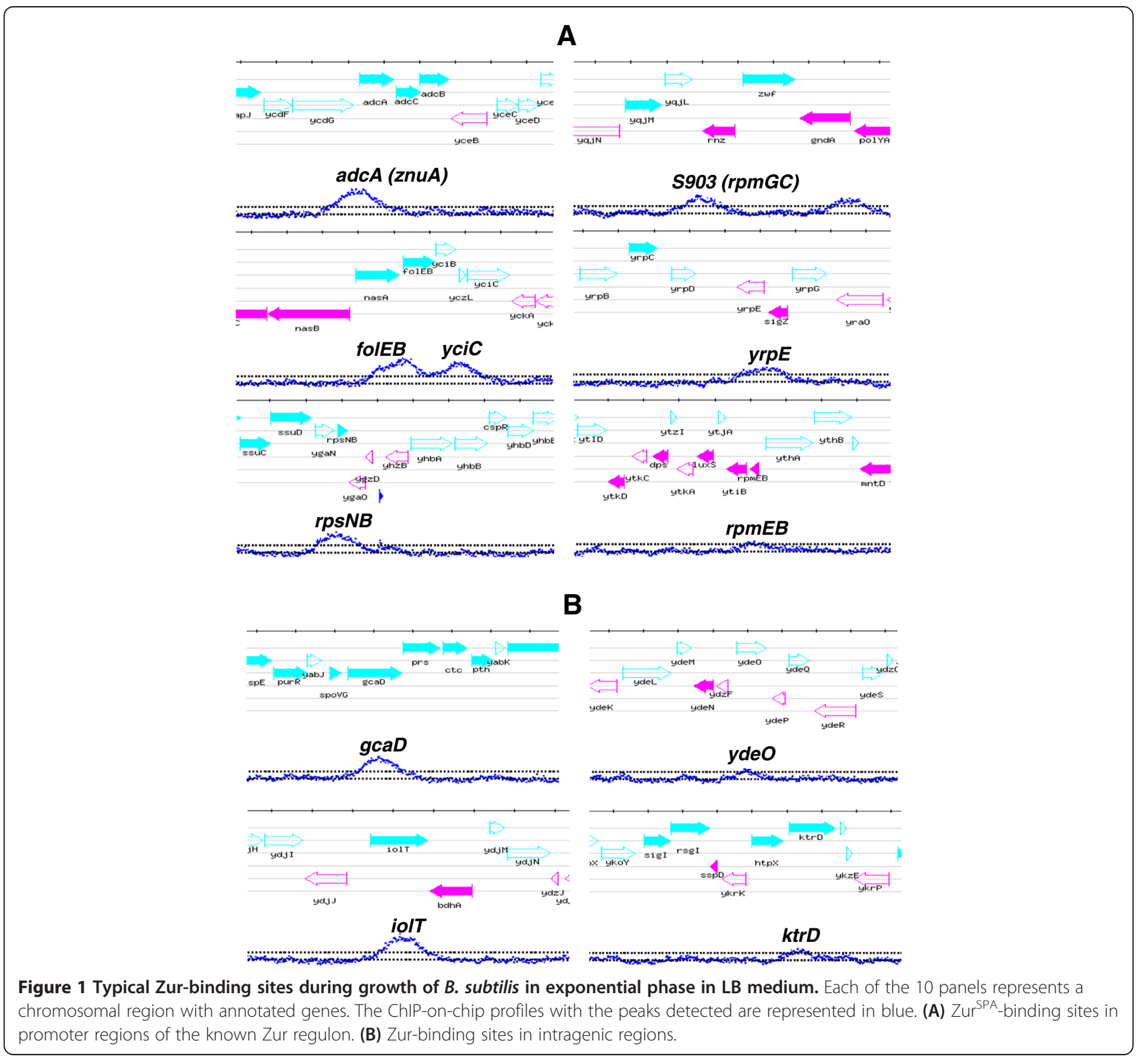


of a translational start site (Additional file 2: Table S1) suggesting a Zur-dependent expression and existence of new candidates to be part of the Zur regulon. The expression of all the genes belonging to the Zur primary regulon displayed a similar expression profile whereas the expression of the genes closed to the 37 newly identified promoterproximal sites did not appear correlated with the Zur primary regulon [26]. These genes might be controlled by Zur under specific unknown conditions. The presence of predicted Zur boxes in these regions is discussed below.

Finally, 35 peaks were located within intragenic regions more than 200 bp downstream a start codon (Figure 1B). The location of these sites was intriguing since none Zur intragenic binding site has so far been described.

\section{Prediction of Zur boxes within Zur-binding sites}

To investigate the presence of Zur boxes within the newly identified Zur-binding sites, we performed in silico analyses. Zur binds to DNA sites, which display high sequence similarity to those recognized by Fur and PerR although regulon overlap in naturally occurring promoter/operator sites has not been reported [27-29]. Here, we used the MEME standard bioinformatic method [30] to identify common motifs among genomic regions representing $100 \mathrm{bp}$ centered at each Zur-binding sites. An appealing feature of this program is its ability to automatically compute optimal motif widths, in contrast to the majority of current motif-finding software. We did not impose a constraint that the motif must be an inverted repeat sequence on the search. This yielded a 20-nt motif present in 40 Zur-binding sites and matching the previously reported Zur box consensus (Figure 2A and B) [25]. The conserved nucleotides at positions 4, 5, 15 and 16 corresponded to the most critical for Zur binding (Figure 2C) [11].

Seven predicted Zur boxes occurred in the promoter regions belonging to the Zur regulon, validating our in silico approach. The 33 newly identified Zur boxes were associated to in vivo Zur binding in inter- as well as in intragenic regions with a broad distribution of ChIPScores (see Methods), which reflect the strength of in vivo Zur-DNA interaction (Additional file 3: Table S2). Correlation between ChIPScores and the degree of similarity of each Zur box to the consensus sequence was investigated. As shown in Figure 3, no clear correlation was observed. It is possible that differences in ChIPScores result not only from Zur-DNA binding affinity but also from differences in cross-linking efficiency at some DNA regions. Interestingly, the highest ChIPScores $(>15)$ were associated to Zur boxes located less than $50 \mathrm{bp}$ from a translational start site (Figure 4) suggesting that the genomic location could contribute to the strength of Zur binding in vivo.

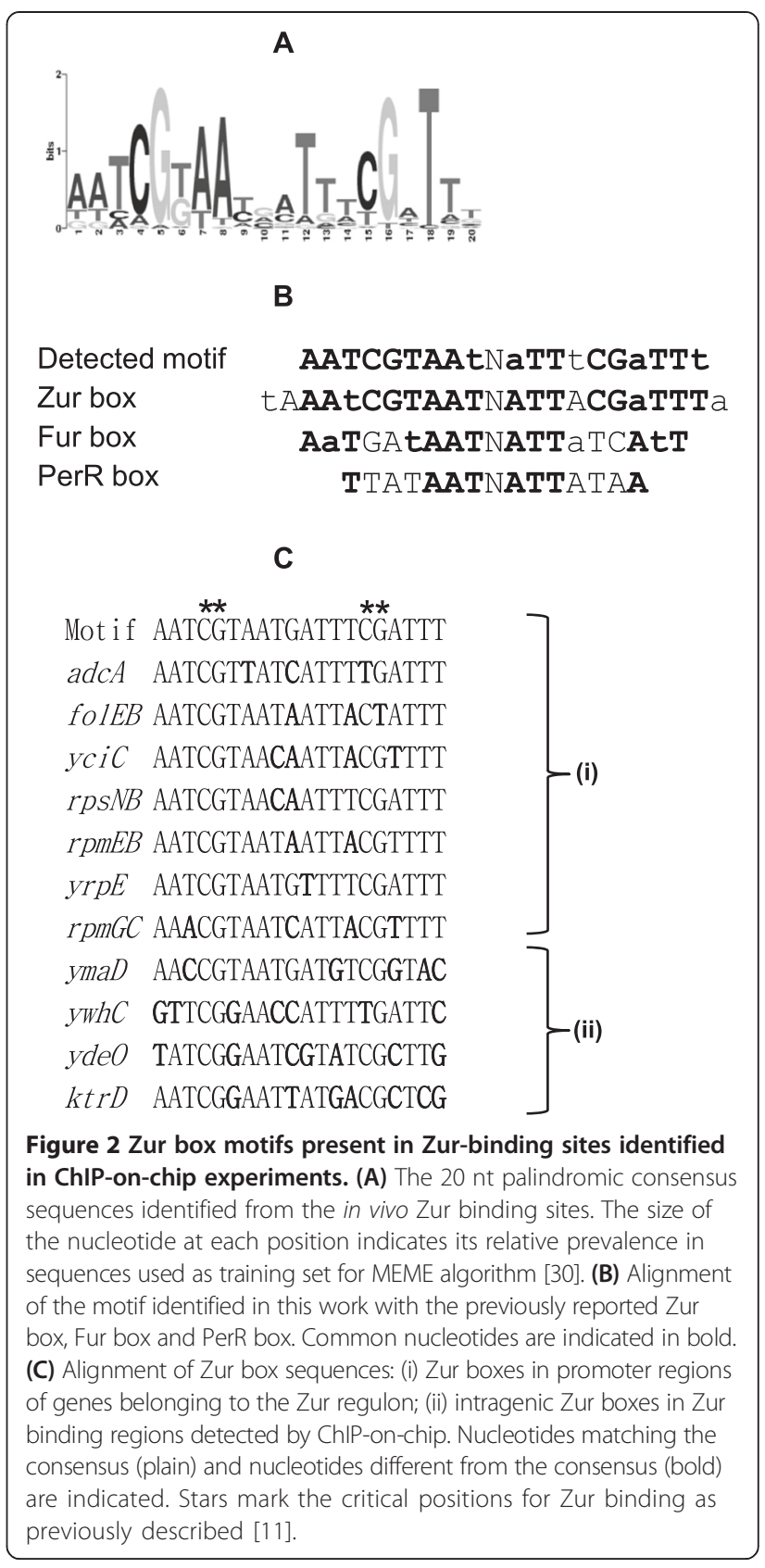

Next, the Zur box consensus was submitted to FIMO [31] to identify putative Zur box in all the B. subtilis genome. Hits with a $p$ value of $\leq 10^{-6}$ were regarded as significant, resulting in 167 candidates including the 40 Zur boxes associated with in vivo Zur binding (see above) (Additional file 4: Table S3). These results showed that only a set of potential Zur boxes were bound by Zur, at least in the conditions used.

Half of the Zur-binding sites detected by ChIP-on-chip did not display a significant match to the Zur box consensus. As shown in Figure 4, these sites were located in inter- as well as in intragenic with some high ChIPScores 


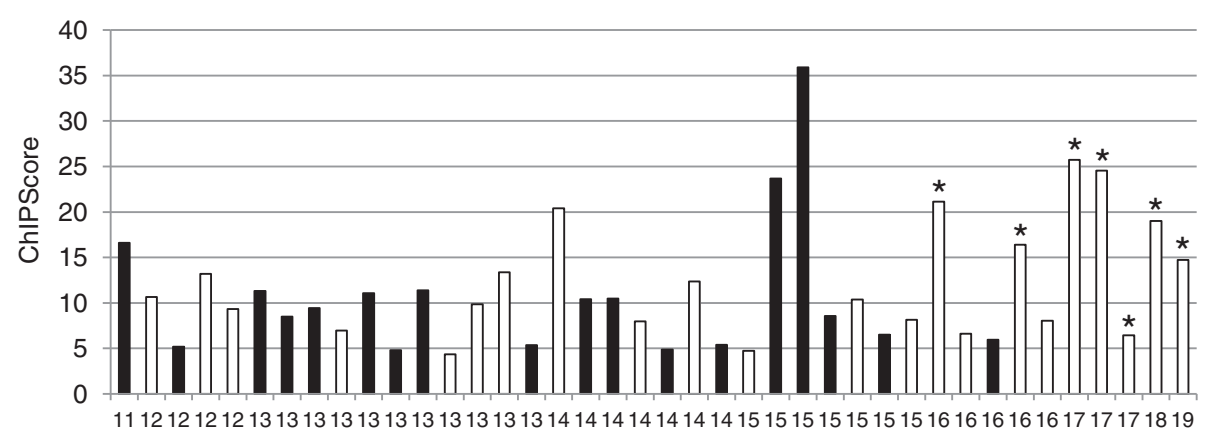

Match to the consensus

Figure 3 Relationship between Zur binding in vivo and similarity to the Zur box consensus. The ChIPScore is scored against the number of nucleotides matching the 20 nt-consensus sequence. Zur box motifs located in intragenic regions (black bars) and Zur box motifs located in intergenic regions (white bars) are indicated. Stars indicate the Zur box motifs belonging to the Zur regulon as previously characterized [10].

(>15). Using MEME, we were unable to identify a common DNA sequence motif among Zur targets that lack a canonical Zur box motif.

\section{The intragenic Zur box from ymaD promotes a Zur-mediated repression when placed within a promoter region}

We further investigated the functionality of an intragenic Zur box to be recognized by the wild-type Zur protein to promote a repressive effect on transcription. We chose the intragenic Zur box located in the ymaD gene encoding a putative peroxiredoxin. Peroxiredoxins are important for antioxidant defense by reducing hydrogen peroxide $\left(\mathrm{H}_{2} \mathrm{O}_{2}\right)$, which induces the zinc uptake system

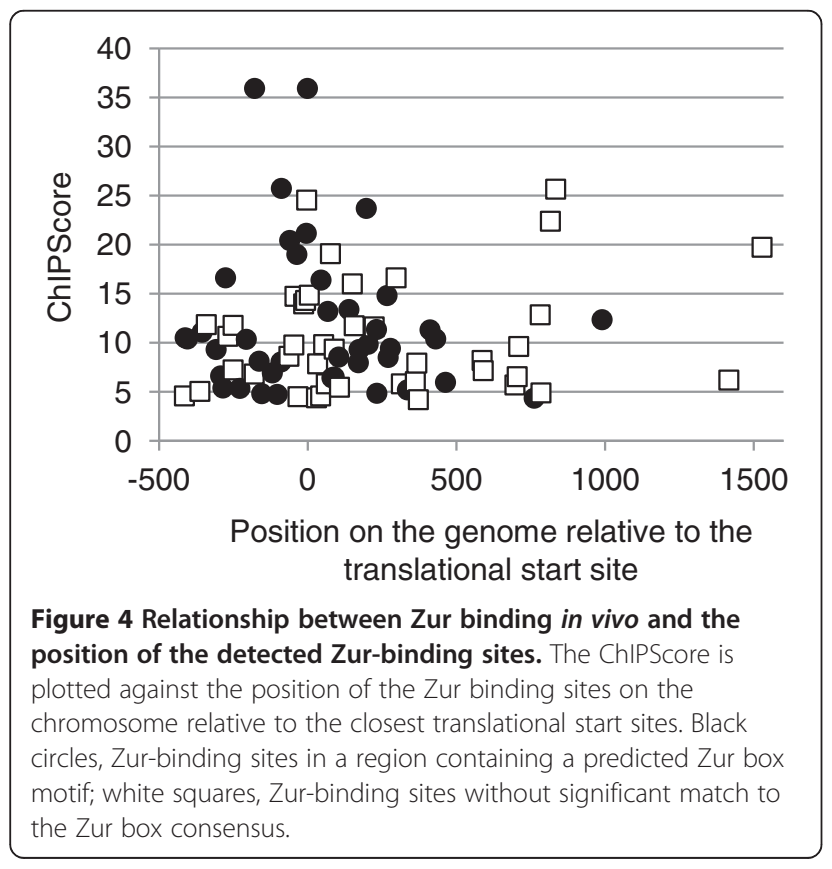

ZosA in B. subtilis [19]. A $\Delta y m a D$ deleted strain (Table 1) was constructed and the sensitivity of this $y m a D$ mutant to $\mathrm{H}_{2} \mathrm{O}_{2}$ was tested in liquid medium. After $30 \mathrm{~min}$ of growth in the presence of $400 \mu \mathrm{M} \mathrm{H}_{2} \mathrm{O}_{2}, 0.005 \%$ and $1 \%$ survival was observed for the mutant and the wild-type cells, respectively (Additional file 5: Figure S2). Thus, deletion of the $y m a D$ gene increased the sensitivity of the cells to $\mathrm{H}_{2} \mathrm{O}_{2}$, indicating that $\mathrm{YmaD}$ plays a role in protecting cells against oxidative stress.

Subsequently, we tested the ability of the ymaD intragenic Zur box to promote Zur-mediated regulation. In a previous in silico prediction, a unique Zur box motif was detected in the $y r p E$ promoter region from nucleotide -22 to -41 relative to the translational start site [25]. $y r p E$ is transcribed from a $\sigma^{\mathrm{A}}$-dependent promoter (Figure 5A) [26]. Two types of transcriptional fusions with the promoter region of the $y r p E$ gene were constructed. First a pAyrpE"-lacZ fusion was obtained using the $y r p E$ promoter sequence spanning from -241 to -1 related to the translational start site, which included the native Zur box sequence. The fusion sequence was integrated at the $a m y E$ locus of the wild-type and $\Delta z$ ur strains (Table 1). Expression of the pAyrpE promoter was 20 -fold increased in a $\Delta z u r$ mutant compared to the wild-type (Figure 5B). In the second pByrpE"-lacZ fusion, the native Zur box of $y r p E$ was replaced by the intragenic Zur box sequence from ymaD (Figure 5A). This fusion exhibited 16-fold higher level in $\Delta z u r$ cells than in the wild-type (Figure 5B). Thus, the intragenic Zur box sequence from $y m a D$ was recognized by Zur to mediate transcriptional repression when located in a promoter region. This confirmed the hypothesis that intragenic Zur box sequences are functional Zur binding sites.

Intragenic Zur boxes: a role in transcription elongation? Some transcription factors, such as B. subtilis CodY and CcpA regulators, can regulate the transcription 


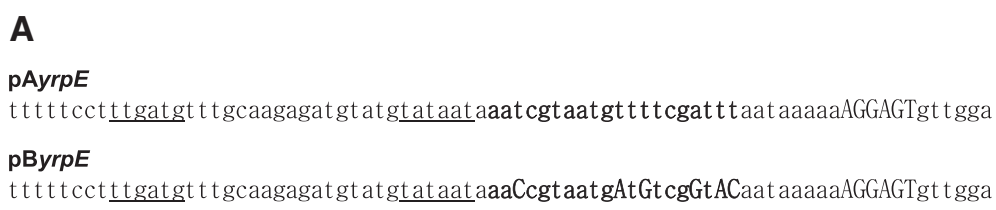

B

$\begin{array}{lllc}\text { pAyrp } E_{-241} & \text { WT } & \text { zur mutant } \\ \text { pByrp } E_{-241} & -43-22-1 & 6 \leq & 104 \pm 11 \\ -43-23^{-1} & 6 \leq & 83 \pm 13\end{array}$

Figure 5 Structure of the $y r p E$ promoter region and expression of $y r p E^{\prime}-l a c Z$ transcriptional fusions. (A) Detailed view of the $y r p E$ promoter region in the two transcriptional fusions. The $\sigma^{\mathrm{A}}$-dependent recognition site is underlined, the ribosome-binding site is capitalized. The Zur box motif is bold. In pAyrpE, the Zur box motif is the native sequence. In pByrpE, the Zur box motif is the intragenic Zur box sequence from $y m a D$. The six nucleotides differences are indicated in bold capital letters. (B) Expression of the transcriptional pAyrpE'-lacZ and pByrpE'-lacZ fusions in the wild-type (WT) strain and the $\Delta$ zur deleted mutant. Nucleotides are numbered relative to the translational start site of the $y r p E$ gene. Location of the Zur motif is indicated by boxes. White box, native Zur box sequence from the yrpE promoter region; black box, Zur box sequence identified within the ymaD gene. $\beta$-galactosidase activities were measured at least 3 times independently.

elongation by a roadblock mechanism [32,33]. Thus, we tested whether several intragenic Zur binding sites could participate in such a regulatory process. The $y d e O$, $y w h C$ and $k t r D$ genes contain intragenic Zur-binding sites and encode putative membrane protein, potential zinc metalloprotease, and $\mathrm{K}^{+}$-transporting ATPase, respectively. They are transcribed from $\sigma^{\mathrm{A}}$-dependent promoters [26]. Different DNA regions were fused to the $l a c Z$ reporter gene and integrated at the $a m y E$ locus of the wild-type strain (Figure 6A, Table 1). The pAymaD, $\mathrm{pA} y d e O, \mathrm{pA} y w h C$ and $\mathrm{pA} k \operatorname{trD}$ fusions contained the promoter region and the start of the encoding-sequence including the native Zur box. The pBymaD, pBydeO, $\mathrm{pB} y w h C$ and $\mathrm{pB} k t r D$ fusions contained symmetric mutations in each half-site of the Zur box at position 4 and 5 (Figure 6A) because these point- mutations were shown to completely abrogate Zur binding (Gabriel et al., [11]). $\beta$-galactosidase activities were examined during exponential growth (Figure 6B). Expression values of the $\mathrm{pAymaD}, \mathrm{pA} y d e O, \mathrm{pA} y w h C$ and $\mathrm{pA} k t r D$ fusions ranged between 2 and 42 units of $\beta$-galactosidase activity. In contrast, the $\mathrm{pB} y m a D, \mathrm{pB} y d e O, \mathrm{pB} y w h C$ and $\mathrm{pB} k t r D$ fusions were 4- to 9-fold upregulated (Figure 6B) suggesting that intragenic Zur boxes mediate a transcriptional cis-repressive effect. Expression of the $\mathrm{pA}$ and $\mathrm{pB}$ fusions was also tested in a zur mutant but similar levels of expression was observed in $\Delta z u r$ and wild-type cells (data not shown).

\section{Zur-binding sites overlap genes responding to disulfide stress}

Our analyses revealed that a large overlap existed between the location of Zur-binding sites and genes whose expression responds to disulfide stress [24]. Zur-binding sites were located less than $400 \mathrm{bp}$ upstream of the translational start site or in the coding sequences of 31 activated and 19-repressed genes in response to diamide stress (Additional file 6: Table S4). Furthermore, Zurbinding sites are associated to 10 genes (citR, cysK, ilvA, $k a t A, S 1408, p p s, y b x G, y m a D, y u s D, y v c I$ ) reported to be directly regulated by Spx [24], suggesting that Zur- and Spx-mediated regulations partially overlap.

To investigate a potential role of an intragenic Zur box to mediate a disulphide stress response, we choose to test the effect of diamide treatment on $y m a D$ expression in wild-type and $\Delta s p x$ genetic backgrounds. As it is known that LacZ activity is very sensitive to diamide addition, we constructed a pAymaD'-luc fusion between the region from nucleotide -268 to +102 relative to $y m a D$ translational start site and the luciferase gene. A second pBymaD'-luc fusion was constructed containing point-mutations in the intragenic Zur box. Luciferase activity was recorded during the growth in LB medium. Expression of pBymaD'-luc was 10-fold higher than expression of pAymaD'-luc (Figure 7A) confirming the results observed with the $l a c Z$ fusions (see above).

No expression of pAymaD'-luc and pBymaD'-luc was detected in a $\Delta s p x$ mutant (Figure 7A), in keeping with the Spx-dependent activation of $y m a D$ [24]. In the wildtype cells, 0.1 or $0.5 \mathrm{mM}$ diamide treatment reproducibly increased pAymaD'-luc expression (Figure 7B). This indicated that Zur binding to the Zur box of pAymaD did not interfere woth Spx activity. The same pattern of induction was observed with pBymaD (Figure 7B). Thus, the intragenic Zur box did not appear involved in the Spx-dependent upregulation.

\section{Discussion}

Using the ChIP-on-chip methodology, we identified 80 enriched DNA-regions in the B. subtilis chromosome 


\section{A}

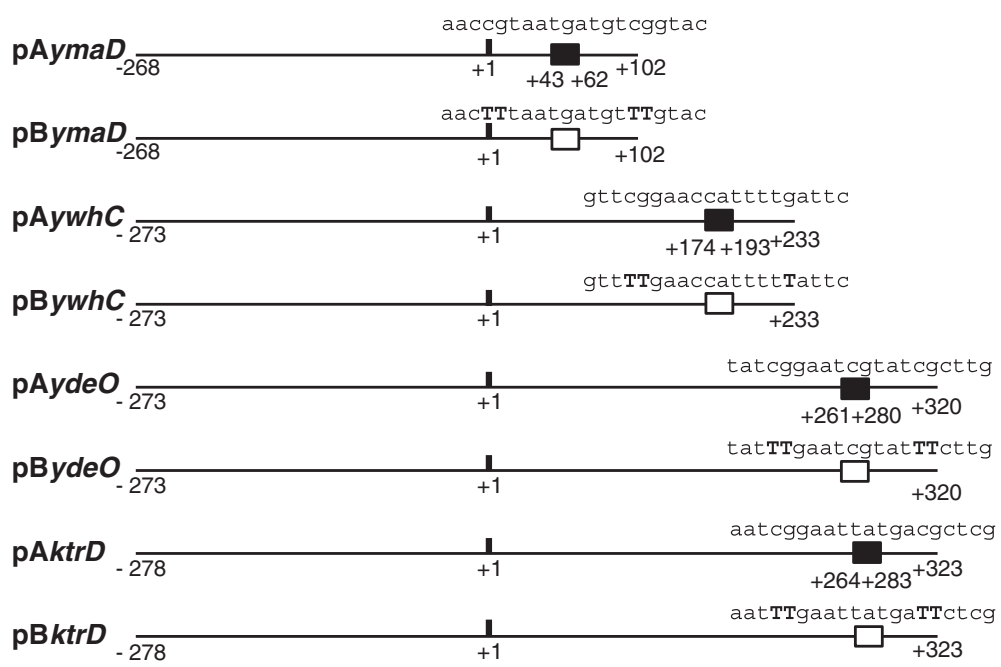

B

Expression assay in the wild-type strain

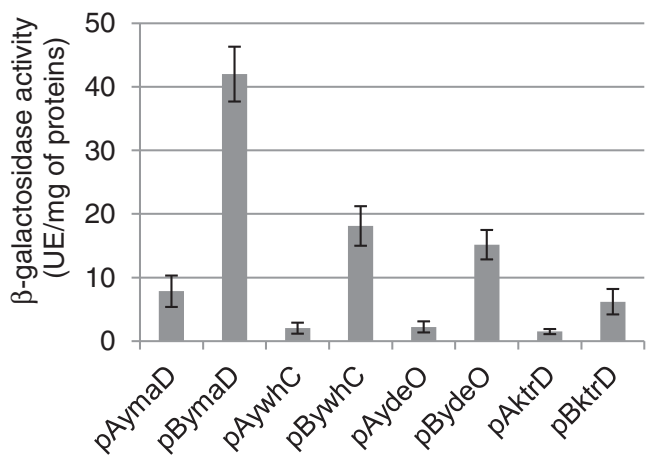

Figure 6 Expression of various transcriptional fusions in the wild-type and the $\boldsymbol{\Delta}$ zur strains. (A) Scheme of the DNA regions inserted upstream from the lacZ reporter gene. Nucleotides are numbered relative to the translational start site of the $y m a D, y w h C, y d e O$ and ktrD genes. The native intragenic Zur boxes are indicated by black boxes. The mutated intragenic Zur boxes are indicated by white boxes. The generated point-mutations in the Zur box sequences are indicated in bold capital letters. (B) Cells were grown in LB medium. $\beta$-galactosidase activities were determined in extracts prepared from exponentially growing cells at $\mathrm{OD}_{600}$ of 0.6 .

that are reproducibly bound by the Zur repressor under abundant zinc growth conditions. We recovered the known Zur regulon and confirmed that the predicted Zur boxes present upstream of yrpE and rpmGC [17,25] are functional binding sites in vivo. These data allow to define the Zur primary regulon, which is now composed of 7 transcription units. Consequently, the whole Zur binding sites identified by ChIP-on-chip appear relevant. Remarkably, a second set of 33 newly identified sites bound by Zur contained a Zur box motif, which differs from the Fur and PerR boxes at positions 4, 5, 15, and 16 and displays conservation of bases at flanking positions that are not strongly conserved in Fur and PerR boxes (positions 1,2,3 and 18, 19, 20) (Figure 2) [11,29]. The last set of Zur binding DNA sites did not contain any direct-repeat sequence or any common motif by the standard bioinformatic methods that we used, suggesting that Zur recognizes degenerated Zur box sequences or that other factors are required for Zur binding at these sites. Interestingly, the Zur binding sites with and without predicted Zur box exhibit a similar distribution relative to coding sequences, with an enrichment around the translation start site (Figure 4), suggesting that both types of sites are functionally similar.

A surprising result from this study was the finding that 35 Zur binding sites are located in intragenic regions. Sixteen of those contain a predicted Zur box. This contrasts sharply with in silico studies, which tend to consider transcription factor-targets within coding sequences as artefacts. Analysis with transcriptional fusions using the 
A
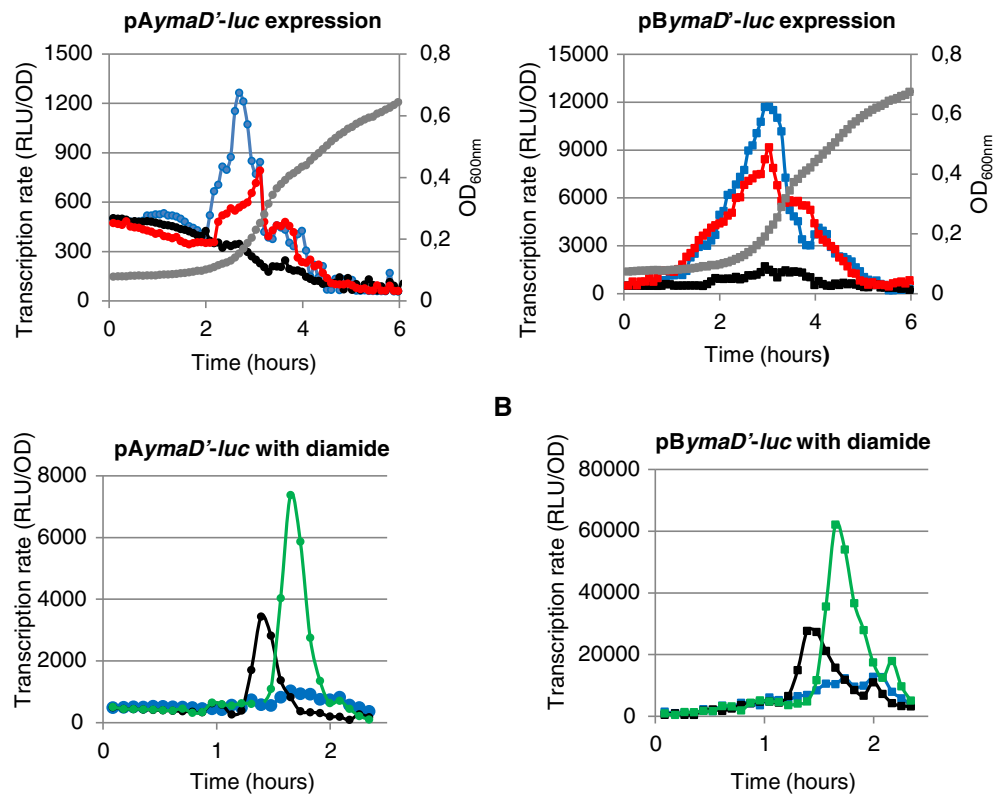

B

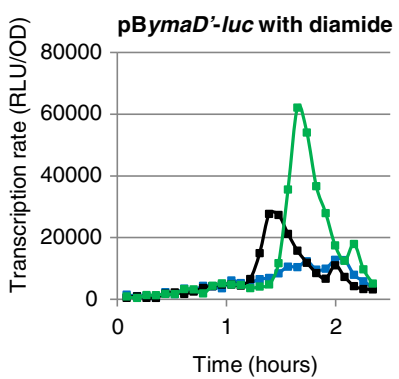

Figure 7 Expression of $y m a D$ in various genetic backgrounds and after diamide treatment. (A) Expression of pAymaD and pBymaD fusions in the wild-type (WT) strain (blue lines), in $\Delta z u r$ (red lines) or $\Delta$ spx cells (black lines). Growth was monitored by measuring the optical density at $600 \mathrm{~nm}$ : grey line, wild-type. The $\Delta z u r$ and $\Delta s p x$ stains grew with the same growth profile than the wild-type. (B) Promoter activity of pAymaD and pBymaD without diamide treatment (blue lines), with $0.1 \mathrm{mM}$ diamide (black lines) or with $0.5 \mathrm{mM}$ diamide (green lines). Final concentration of 0.1 or $0.5 \mathrm{mM}$ diamide was added at $\mathrm{t}=1$ hour when cells reached an $\mathrm{OD}_{600}$ of $0.2 \mathrm{in} \mathrm{LB}$ medium. For each strain, one representative curve, out of three independent replicates realized, is shown.

lac $Z$ reporter gene allowed us to show that the intragenic Zur box sequence from the $y m a D$ gene was fully functional for Zur-mediated repression when placed in a promoter region. Thus, the experimental strategies used in this study revealed unexpected and functional Zur binding sites. In addition, we showed that the intragenic Zur box within $y m a D, y d e O, y w h C$ and $k t r D$ genes had a cis-repressive effect (3- to 10-fold) on transcription. Expression of these genes was also tested in a $\Delta z u r$ mutant but similar levels of expression was observed in $\Delta z u r$ and wild-type cells (data not shown). As disruption of zinc homeostasis in $\Delta z u r$ cells likely affects many cellular processes, the direct role of Zur binding to the intragenic Zur boxes could be masked by other regulatory effects.

Our study highlights the presence of Zur boxes within a subset of genes encoding functions related to metal ion homeostasis or oxidative stress. We showed that the ymaD gene codes for putative peroxiredoxin-related protein, which plays a role in protecting cells against oxidative stress. Interestingly, ymaD is also under direct regulation by Spx [24] a global transcription key regulator for maintaining redox homeostasis of B. subtilis cells exposed to disulfide stress [34]. The intragenic Zur box of $y m a D$ did not appear to play a direct role on the Spxdependent regulation. Remarkably, we observed that the $y m a D$ transient induction upon exposure to diamide was abolished in $\Delta z u r$ cells (data not shown) pointing to an interconnection of the Zur- and Spx-mediated responses. As the degradation of Spx is ensured by the ClpXP protease [35], disruption of zinc homeostasis in $\Delta z u r$ cells could impact on the activity of the $\mathrm{Zn}$ dependent protein ClpX and, as a consequence, on the turnover of Spx. Overall, it was not possible to conclude about the direct role of Zur to mediate a regulation via the intragenic Zur boxes because of $\Delta z u r$ deletion may entail significantly changes in other transcription factors activity. In this intricate regulatory network, the binding of Zur to intragenic Zur boxes may contribute to finetune gene expression in response to zinc availability.

The interconnection between Zur and Spx may involve more than one gene as Zur binding sites were detected within or near $10 \mathrm{Spx}$-regulated genes and overall near 50 genes responsive to diamide stress (Additional file 6: Table S4). Overlap between disulfide and oxidative stress responses was previously identified in $B$. subtilis for the katA gene encoding a catalase. Expression of katA is under the dual control of PerR [29] and Spx [24] regulators. In addition, a regulatory interplay between the responses to zinc deprivation and disulfide stress has been described in Streptomyces coelicolor, where the activity of the thiol-disulfide metabolism regulator $\sigma^{R}$ is induced upon zinc limitation [36]. Our results emphasize the 
complex interplay between the regulatory networks controlling zinc homeostasis and redox homeostasis, especially the oxidative and disulfide stress responses.

\section{Conclusions}

The Chip-on-chip approach used in this study allowed to considerably expand the catalogue of in vivo Zurbinding sites to 80 inter- as well as intragenic regions. Half of those is associated with an in silico predicted Zur box. The binding of Zur to the newly identified targets may contribute to fine-tune gene expression under specific conditions, our results highlighting a complex link between Zur and the disulfide stress response. Intragenic Zur boxes could be involved in an intricate mechanisms of regulation of the transcription elongation, possibly with other transcriptional factors. Future investigations will be required to investigate the role of Zur binding sites in transcriptional regulation.

\section{Methods}

\section{Bacterial strains and growth conditions}

The B. subtilis strains used in this work are listed in Table 1. E. coli and B. subtilis cells were grown in LuriaBertani (LB) medium or in MS medium containing $62 \mathrm{mM} \mathrm{K}_{2} \mathrm{HPO}_{4}, 44 \mathrm{mM} \mathrm{KH}_{2} \mathrm{PO}_{4}, 17 \mathrm{mM}$ trisodium citrate, $11 \mathrm{mM} \mathrm{K}_{2} \mathrm{SO}_{4}, 0.4 \%$ glucose, $0.06 \%$ L-glutamine, $0.01 \%$ L-tryptophan, $0.1 \%$ casamino acids, $1 \mathrm{mM} \mathrm{MgSO}_{4}$, $1 \mathrm{mM} \mathrm{CaCl}_{2}, 100 \mu \mathrm{M} \mathrm{FeCl}_{3}$ citrate, $112 \mu \mathrm{M} \mathrm{ZnCl}_{2} ; 5 \mu \mathrm{M}$ $\mathrm{MnCl}_{2} ; 2.5 \mu \mathrm{M} \mathrm{CuCl}_{2}$. Antibiotics were added at the following concentrations when required: $100 \mu \mathrm{g}$ ampicillin $\mathrm{ml}^{-1}$; $5 \mu \mathrm{g}$ kanamycin $\mathrm{ml}^{-1}$; $10 \mu \mathrm{g}$ erythromycin $\mathrm{ml}^{-1}$; $5 \mu \mathrm{g}$ chloramphenicol ml $\mathrm{ml}^{-1} ; 60 \mu \mathrm{g}$ spectinomycin $\mathrm{ml}^{-1}$. Solid media were prepared by addition of $20 \mathrm{~g}$ Agar noble $\mathrm{l}^{-1}$ (Difco). Standard procedures were used to transform E. coli [37] and B. subtilis [38].

\section{DNA manipulations}

DNA manipulations and cloning procedures were performed as described elsewhere [37] according to standard procedures. Restriction enzymes, Pfu DNA polymerase and phage T4 DNA ligase were used as recommended by the manufacturer (Biolabs). DNA fragments were purified from agarose gels using the QIAquick kit (Qiagen).

\section{Construction of plasmids and strains}

A B. subtilis strain expressing a C-terminal SPA-tagged Zur protein (hereafter Zur $^{\mathrm{SPA}}$ ) was constructed by chromosomal integration of a translational fusion between the zur coding sequence and the sequential peptide affinity (SPA) tag sequence $[39,40]$, resulting in the BSAS36 strain expressing Zur ${ }^{\mathrm{SPA}}$ under the control of its native promoter as unique source of Zur. In this purpose, the $z u r$ coding sequence (from nucleotide +13 to +435 relative to the translational start site) was amplified by
PCR with oligonucleotides creating an Acc651 restriction site at the $5^{\prime}$ end (5'-GGAATTGGTACCgaagcgctgaacc tattaaaa-3') and a NcoI restriction site at the $3^{\prime}$ end of the fragment (5'-GGAATTCCATGGcgcagtagtgttt tcttggtt-3'). The PCR product was cloned into plasmid pMUTIN-SPA subsequent to digestion with Acc651 and NcoI [41]. The resulting plasmid was used to transform B. subtilis and to select for erythromycin-resistance. Integration was confirmed by PCR and verified by DNA sequencing.

The zur mutant BSAS45 was constructed by homologous replacement of the Zur coding sequence with the kanamycin-resistance gene aphA3 using a joining PCR technique [42]. The aphA3 gene was first amplified. The region upstream of the zur gene (nucleotides -887 to +65 relative to the translational start site) was amplified by PCR with a 21 bp aphA3 fragment at its 3' end. The region downstream of zur (nucleotides +366 to +1321 ) was amplified with a 21 bp aphA3 fragment at its $5^{\prime}$ end. The three DNA fragments were combined and then a PCR reaction was performed with the two external oligonucleotides. The final product, corresponding to the two regions flanking zur with the inserted aphA3 cassette in between, was purified from a gel and used to transform B. subtilis. Integration and deletion were confirmed by PCR and verified by DNA sequencing. The ymaD mutant BSAS239 was constructed by the same strategy. The region upstream of the ymaD gene (nucleotides -921 to +90 relative to the translational start site) was amplified by PCR with a 21 bp aphA3 fragment at its 3' end. The region downstream of ymaD (nucleotides +400 to +1431 ) was amplified with a 21 bp aphA3 fragment at its $5^{\prime}$ end. The joining PCR, corresponding to the two regions flanking ymaD with the inserted aphA3 cassette in between was used to transform $B$. subtilis.

The spx mutant BSAS296 was constructed by homologous replacement of the Spx coding sequence with a spectinomycin-resistance gene $s p c$. The $s p c$ gene was first amplified. The region upstream of the $s p x$ gene (nucleotides -861 to +57 relative to the translational start site) was amplified by PCR with a $21 \mathrm{bp} s p c$ fragment at its 3 ' end. The region downstream of $s p x$ (nucleotides +348 to +1270 ) was amplified with a $21 \mathrm{bp}$ $s p c$ fragment at its $5^{\prime}$ end. The three DNA fragments were combined and then a PCR reaction was performed with the two external oligonucleotides. The final product, corresponding to the two regions flanking $s p x$ with the inserted $s p c$ cassette in between, was purified from a gel and used to transform B. subtilis. Integration and deletion were confirmed by PCR and verified by DNA sequencing.

To construct transcriptional fusions with the lac $Z$ reporter gene, DNA fragments corresponding to the various promoter regions under investigation were amplified by PCR. Oligonucleotides were used to create an EcoRI 
restriction site at the $5^{\prime}$ end and a $\mathrm{BamHI}$ restriction site at the $3^{\prime}$ end of the fragments. PCR products were cloned into plasmid pAC6 subsequent to digestion with EcoRI and BamHI [43]. In this way, the promoter region of $y c i C$ (from nucleotide -312 to -1 relative to the translational start site) was fused with the lac $Z$ reporter gene. The transcriptional fusions with the $l a c Z$ gene were subsequently integrated at the $a m y E$ locus of $B$. subtilis (Table 1). To generate pBymaD, pBydeO, $\mathrm{pB} y w h C$ and $\mathrm{pB} k t r D$ fusions, we used large primers introducing point-mutations in the Zur box motifs (Figure 6A). The resulting constructs were verified by DNA sequencing. $\beta$-galactosidase specific activities were measured during exponential phase growth in LB medium, as described by Miller with cell extracts obtained by lysozyme treatment [44]. One unit of $\beta$-galactosidase activity was defined as the amount of enzyme that produces $1 \mathrm{nmol}$ $o$-nitrophenol min- 1 at $28^{\circ} \mathrm{C}$. The mean values and standard deviations of at least three independent experiments are shown.

To construct transcriptional fusions with the luc reporter gene, we used the assembly Gibson's procedure [45] to obtain transcriptional fusions with luc instead of the lacZ gene. The PUC18cm-luc plasmid [46] was used as template to amplify the luc reporter gene. The sequence of the resulting constructs were verified by DNA sequencing. The mean values and standard deviations of at least three independent experiments are shown.

\section{Luciferase assay}

For the detection of luciferase activity, strains were first grown in LB medium to an optical density at $600 \mathrm{~nm}$ $\left(\mathrm{OD}_{600}\right)$ of 2 . Cells were then centrifuged and resuspended in fresh LB medium, adjusting all the cultures to an $\mathrm{OD}_{600}$ of 1 . These pre-cultures were then diluted 20 fold in fresh LB medium and $200 \mu \mathrm{l}$ was distributed in each of two wells in a 96-well black plate (Corning). $10 \mu \mathrm{l}$ of luciferin were added to each well to reach a final concentration of $1.5 \mathrm{mg} / \mathrm{ml}(4.7 \mathrm{mM})$. The cultures were incubated at $37^{\circ} \mathrm{C}$ with agitation in a PerkinElmer Envision 2104 Multilabel Reader equipped with an enhanced sensitivity photomultiplier for luminometry. The temperature of the clear plastic lid was maintained at $38^{\circ} \mathrm{C}$ to avoid condensation. Relative Luminescence Unit (RLU) and $\mathrm{OD}_{600}$ were measured at 5 min intervals.

\section{Genome-wide determination of the Zur-binding sites by ChIP-on-chip}

Chromatin Immnunoprecipitation assays were performed to measure the chromosome-wide DNA-binding profiles of Zur, as described previously [26]. Briefly, strain BSAS36 was cultivated at $37^{\circ} \mathrm{C}$ until an $\mathrm{OD}_{600}$ of 0.6 in $\mathrm{LB}$ medium with $1 \mu \mathrm{g}$ erythromycin $\mathrm{ml}^{-1}$. Cells were treated with formaldehyde, cellular DNA was extracted and sonicated, and an antibody against the SPA-tag was used to preferentially purify the DNA regions specifically cross-linked to Zur $^{\text {SPA. }}$. The immuno-precipitated DNA (IP) and the control whole cell DNA extract (WCE) were labeled with Cy3 and Cy5, respectively, and co-hybridized to the B. subtilis Roche-NimbleGen tiled microarrays [47].

\section{Peak sequence extraction and analysis}

Identification of peaks corresponding to chromosomal Zur binding sites was performed as described in [48]. IP/ WCE ratios $(\log 2)$ were corrected for dye bias using Loess regression on the MA plot. The signal was smoothed by two rounds of sliding window averaging (29 probes, around $320 \mathrm{bp}$ ). Maxima (or minima) were defined as probes for which the smoothed signal is the highest (or lowest respectively) into the window used for smoothing. Peaks within the same 300 bp window were merged. The peak height was calculated as the $\log 2$ ratio difference between the smoothed signal values of the maxima and the adjacent minima. In order to quantify enrichment of Zur-bound DNA regions, the signal was smoothed and a ChipScore was calculated as described by Buescher et al. [23]. Briefly, this score is based on the distribution of the peak height values and estimates for each peak its relative distance from the median $($ ChipScore $=[$ height-median $] /[$ upperquartile-median $]$ ). Only the regions associated with a peak scoring $\geq 4$ [a threshold determined empirically from ChIP-on-chip experiments with the transcription factor CcpA [23]] in both replicates were considered as putative Zur-binding sites in the subsequent analyses.

\section{Availability}

The data discussed in this publication have been deposited in NCBI's Gene Expression Omnibus and are accessible through GEO Series accession number GSE64671 (http:// www.ncbi.nlm.nih.gov/geo/query/acc.cgi?acc=GSE64671).

\section{Additional files}

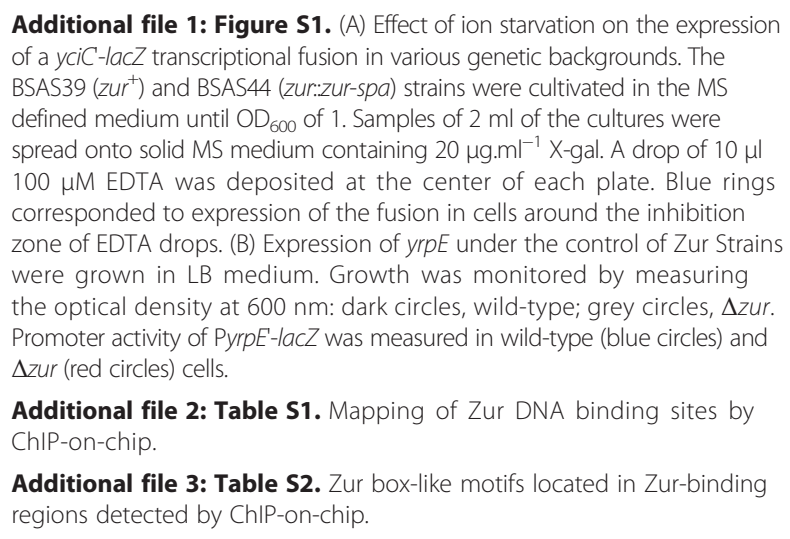

Additional file 2: Table S1. Mapping of Zur DNA binding sites by ChIP-on-chip.

Additional file 3: Table S2. Zur box-like motifs located in Zur-binding regions detected by ChIP-on-chip. 
Additional file 4: Table S3. FIMO analysis of the occurrence of the Zur box motif in the whole B. subtilis genome.

Additional file 5: Figure S2. Comparison of the effect of hydrogen peroxide on the survival of $\triangle y m a D$ and wild-type cells. Survival of BSAS239 $\triangle y m a D$ (open triangles) and wild-type (black triangles) growing cells in LB medium were calculated after 15 and 30 min of challenge with $400 \mu \mathrm{M} \mathrm{H}_{2} \mathrm{O}_{2}$. Hydrogen peroxide was added at $\mathrm{OD}_{600}$ of 0.6. One hundred per cent corresponds to the number of c.f.u. before $\mathrm{H}_{2} \mathrm{O}_{2}$ was applied (approx $5.10^{7}$ c.f.u. $\mathrm{ml}^{-1}$ of each culture).

Additional file 6: Table S4. Overlap between Zur binding-sites and response to diamide stress.

\section{Competing of interests}

The authors declare that they have no competing interests.

\section{Authors' contributions}

EP and SA conceived and designed the experiments, analyzed the data and drafted the manuscript. PN participated in the design of the study and helped to draft the manuscript. All authors read and approved the final manuscript.

\section{Acknowledgments}

We would like to thank Dr Etienne Dervyn for the help with transcriptomic analyses and Dr Nathalie Pigeonneau for the help with ChIP-on-chip data analyses. We are grateful to Dr Elena Bidnenko and Dr Olivier Delumeau for careful reading of the manuscript.

Received: 23 September 2014 Accepted: 13 January 2015 Published online: 04 February 2015

\section{References}

1. McCall KA, Huang CC, Fierke CA. Function and Mechanism of Zinc Metalloenzymes. J Nutr. 2000;130(5):1437S-46.

2. Krishna SS, Majumdar I, Grishin NV. Structural classification of zinc fingers. Nucleic Acids Res. 2003;31(2):532-50.

3. McDevitt CA, Ogunniyi AD, Valkov E, Lawrence MC, Kobe B, McEwan AG, et al. A molecular mechanism for bacterial susceptibility to zinc. PLoS Pathog. 2011;7(11):e1002357.

4. Blencowe DK, Morby AP. Zn(II) metabolism in prokaryotes. FEMS Microbiol Rev. 2003:27(2-3):291-311.

5. Moore CM, Gaballa A, Hui M, Ye RW, Helmann JD. Genetic and physiological responses of Bacillus subtilis to metal ion stress. Mol Microbiol. 2005:57(1):27-40.

6. Patzer SI, Hantke K. The ZnuABC high-affinity zinc uptake system and its regulator Zur in Escherichia coli. Mol Microbiol. 1998;28(6):1199-210.

7. Gaballa A, Helmann JD. Identification of a zinc-specific metalloregulatory protein, Zur, controlling zinc transport operons in Bacillus subtilis. J Bacteriol. 1998;180(22):5815-21.

8. Outten CE, Tobin DA, Penner-Hahn JE, O'Halloran TV. Characterization of the metal receptor sites in Escherichia coli Zur, an ultrasensitive zinc(II) metalloregulatory protein. Biochemistry. 2001;40(35):10417-23.

9. Lee JW, Helmann JD. Functional specialization within the Fur family of metalloregulators. Biometals. 2007;20(3-4):485-99. Epub 2007 Jan 2010

10. Gaballa A, Wang T, Ye RW, Helmann JD. Functional analysis of the Bacillus subtilis Zur regulon. J Bacteriol. 2002;184(23):6508-14.

11. Gabriel SE, Miyagi F, Gaballa A, Helmann JD. Regulation of the Bacillus subtilis yciC gene and insights into the DNA-binding specificity of the zinc-sensing metalloregulator Zur. J Bacteriol. 2008;190(10):3482-8.

12. Ma Z, Gabriel SE, Helmann JD. Sequential binding and sensing of Zn(II) by Bacillus subtilis Zur. Nucleic Acids Res. 2011;39(21):9130-8.

13. Sankaran B, Bonnett SA, Shah K, Gabriel S, Reddy R, Schimmel P, et al. Zinc independent folate biosynthesis: genetic, biochemical, and structural investigations reveal new metal dependence for GTP cyclohydrolase IB. J Bacteriol. 2009;191(22):6936-49.

14. Nanamiya H, Akanuma G, Natori Y, Murayama R, Kosono S, Kudo T, et al. Zinc is a key factor in controlling alternation of two types of $L 31$ protein in the Bacillus subtilis ribosome. Mol Microbiol. 2004:52(1):273-83.

15. Akanuma G, Nanamiya H, Natori $Y$, Nomura N, Kawamura F. Liberation of zinc-containing L31 (RpmE) from ribosomes by its paralogous gene product, YtiA, in Bacillus subtilis. J Bacteriol. 2006;188(7):2715-20.
16. Nanamiya $H$, Kawamura F. Towards an elucidation of the roles of the ribosome during different growth phases in Bacillus subtilis. Biosci Biotechnol Biochem. 2010;74(3):451-61.

17. Gabriel SE, Helmann JD. Contributions of Zur-controlled ribosomal proteins to growth under zinc starvation conditions. J Bacteriol. 2009:191(19):6116-22.

18. Natori $Y$, Nanamiya H, Akanuma G, Kosono S, Kudo T, Ochi K, et al. A fail-safe system for the ribosome under zinc-limiting conditions in Bacillus subtilis. Mol Microbiol. 2007;63(1):294-307.

19. Gaballa A, Helmann JD. A peroxide-induced zinc uptake system plays an important role in protection against oxidative stress in Bacillus subtilis. Mol Microbiol. 2002;45(4):997-1005.

20. Ogura M. ZnuABC and ZosA zinc transporters are differently involved in competence development in Bacillus subtilis. J Biochem. 2011;150(6):615-25.

21. Moore CM, Helmann JD. Metal ion homeostasis in Bacillus subtilis. Curr Opin Microbiol. 2005;8(2):188-95.

22. Michna RH, Commichau FM, Todter D, Zschiedrich CP, Stulke J. SubtiWiki-a database for the model organism Bacillus subtilis that links pathway, interaction and expression information. Nucleic Acids Res. 2013:42:D692-8.

23. Buescher JM, Liebermeister W, Jules M, Uhr M, Muntel J, Botella E, et al. Global network reorganization during dynamic adaptations of Bacillus subtilis metabolism. Science. 2012;335:1099-103.

24. Rochat T, Nicolas P, Delumeau O, Rabatinova A, Korelusova J, Leduc A, et al, Genome-wide identification of genes directly regulated by the pleiotropic transcription factor Spx in Bacillus subtilis. Nucleic Acids Res. 2012;40 (19):9571-83.

25. Panina EM, Mironov AA, Gelfand MS. Comparative genomics of bacterial zinc regulons: enhanced ion transport, pathogenesis, and rearrangement of ribosomal proteins. Proc Natl Acad Sci. 2003;100(17):9912-7.

26. Nicolas P, Mader U, Dervyn E, Rochat T, Leduc A, Pigeonneau N, et al. Condition-dependent transcriptome reveals high-level regulatory architecture in Bacillus subtilis. Science. 2012:335:1103-6.

27. Baichoo N, Wang T, Ye R, Helmann JD. Global analysis of the Bacillus subtilis Fur regulon and the iron starvation stimulon. Mol Microbiol. 2002;45(6):1613-29.

28. Fillat MF. The FUR (ferric uptake regulator) superfamily: diversity and versatility of key transcriptional regulators. Arch Biochem Biophys. 2014:546:41-52.

29. Fuangthong M, Helmann JD. Recognition of DNA by three ferric uptake regulator (Fur) homologs in Bacillus subtilis. J Bacteriol. 2003;185(21):6348-57.

30. Bailey TL, Williams N, Misleh C, Li WW. MEME: discovering and analyzing DNA and protein sequence motifs. Nucleic Acids Res. 2006;34:W369-73.

31. Grant CE, Bailey TL, Noble WS. FIMO: scanning for occurrences of a given motif. Bioinformatics. 2011;27(7):1017-8.

32. Choi SK, Saier Jr MH. Regulation of sigL expression by the catabolite control protein CcpA involves a roadblock mechanism in Bacillus subtilis: potential connection between carbon and nitrogen metabolism. J Bacteriol. 2005;187(19):6856-61.

33. Belitsky BR, Sonenshein AL. Roadblock repression of transcription by Bacillus subtilis CodY. J Mol Biol. 2011;411:729-43.

34. Nakano S, Erwin KN, Ralle M, Zuber P. Redox-sensitive transcriptional control by a thiol/disulphide switch in the global regulator, Spx. Mol Microbiol. 2005;55(2):498-510.

35. Zhang $Y$, Zuber P. Requirement of the zinc-binding domain of ClpX for Spx proteolysis in Bacillus subtilis and effects of disulfide stress on ClpXP activity. J Bacteriol. 2007;189(21):7669-80

36. Owen GA, Pascoe B, Kallifidas D, Paget MS. Zinc-responsive regulation of alternative ribosomal protein genes in Streptomyces coelicolor involves zur and sigmaR. J Bacteriol. 2007;189(11):4078-86.

37. Sambrook J, Fristch EF, Maniatis T, editors. Molecular cloning: a laboratory manual. Cold Spring Harbor: Cold Spring Harbor Press; 1989.

38. Kunst F, Rapoport G. Salt stress is an environmental signal affecting degradative enzyme synthesis in Bacillus subtilis. J Bacteriol. 1995;177(9):2403-7.

39. Butland G, Peregrin-Alvarez JM, Li J, Yang W, Yang X, Canadien V, et al. Interaction network containing conserved and essential protein complexes in Escherichia coli. Nature. 2005;433(7025):531-7.

40. Zeghouf M, Li J, Butland G, Borkowska A, Canadien V, Richards D, et al Sequential Peptide Affinity (SPA) system for the identification of mammalian and bacterial protein complexes. J Proteome Res. 2004;3(3):463-8. 
41. Lecointe F, Serena C, Velten M, Costes A, McGovern S, Meile JC, et al. Anticipating chromosomal replication fork arrest: SSB targets repair DNA helicases to active forks. Embo J. 2007;26(19):4239-51.

42. Wach A. PCR-synthesis of marker cassettes with long flanking homology regions for gene disruptions in S. cerevisiae. Yeast. 1996;12(3):259-65.

43. Stülke J, Martin-Verstraete I, Zagorec M, Rose M, Klier A, Rapoport G. Induction of the Bacillus subtilis ptsGHI operon by glucose is controlled by a novel antiterminator, GlcT. Mol Microbiol. 1997;25(1):65-78.

44. Miller JH. Assay of B-galactosidase. Cold Spring Harbor: Cold Spring Harbor Laboratory; 1972.

45. Gibson DG, Young L, Chuang RY, Venter JC, Hutchison 3rd CA, Smith HO Enzymatic assembly of DNA molecules up to several hundred kilobases. Nat Methods. 2009;6(5):343-5.

46. Mirouze N, Prepiak P, Dubnau D. Fluctuations in spo0A transcription control rare developmental transitions in Bacillus subtilis. PLoS Genet. 2011;7(4):e1002048.

47. Rasmussen $\mathrm{S}$, Nielsen $\mathrm{HB}$, Jarmer $\mathrm{H}$. The transcriptionally active regions in the genome of Bacillus subtilis. Mol Microbiol. 2009;73:1043-57.

48. Reppas NB, Wade JT, Church GM, Struhl K. The transition between transcriptional initiation and elongation in E. coli is highly variable and often rate limiting. Mol Cell. 2006;24:747-57.

\section{Submit your next manuscript to BioMed Central and take full advantage of:}

- Convenient online submission

- Thorough peer review

- No space constraints or color figure charges

- Immediate publication on acceptance

- Inclusion in PubMed, CAS, Scopus and Google Scholar

- Research which is freely available for redistribution 